\title{
An in vivo molecular response analysis of colorectal cancer treated with Astragalus membranaceus extract
}

\author{
AILUN TSENG ${ }^{1}$, CHIH-HSUEH YANG ${ }^{2}$, CHIH-HAO CHEN $^{1}$, CHANG-HAN CHEN $^{3}$, SHIH-LAN HSU ${ }^{4}$, \\ MEI-HSIEN LEE ${ }^{5}$, HOONG-CHIEN LEE ${ }^{6-10}$ and LI-JEN SU ${ }^{1,11,12}$ \\ ${ }^{1}$ Institute of Systems Biology and Bioinformatics, National Central University, Taoyuan $320 ;{ }^{2}$ Graduate Institute of
Microbiology and Public Health, National Chung Hsing University, Taichung $402 ;{ }^{3}$ Institute for Translational Research
in Biomedicine, Kaohsiung Chang Gung Memorial Hospital, Kaohsiung $833 ;{ }^{4}$ Department of Education and Research,
Taichung Veterans General Hospital, Taichung $407 ;{ }^{5}$ Graduate Institute of Pharmacognosy, College of Pharmacy,
Taipei Medical University, Taipei $110 ;{ }^{6}$ Center for Dynamical Biomarkers and Translational Medicine, National
Central University, Taoyuan $320 ;{ }^{7}$ Division of Hematology and Oncology, Cathay General Hospital, Taipei 106;
${ }^{8}$ Department of Physics, Chung Yuan Christian University, Taoyuan $320 ;{ }^{9}$ Cathay Medical Research Institute,
Cathay General Hospital, Taipei $106 ;{ }^{10}$ Physics Division, National Center for Theoretical Sciences, Hsinchu $300 ;$
${ }^{11}$ Core Facilities for High Throughput Experimental Analysis of Institute of Systems Biology and Bioinformatics,
National Central University, Taoyuan $320 ;{ }^{12}$ IHMED Reproductive Medical Center, Taipei 106 , Taiwan, R.O.C.
}

Received August 7, 2015; Accepted September 4, 2015

DOI: $10.3892 /$ or.2015.4441

\begin{abstract}
The fact that many chemotherapeutic drugs cause chemoresistance and side effects during the course of colorectal cancer treatment necessitates development of novel cytotoxic agents aiming to attenuate new molecular targets. Here, we show that Astragalus membranaceus (Fischer) Bge. var. mongolicus (Bge.) Hsiao (AM), a traditional Chinese medicine, can inhibit tumor growth in vivo and elucidate the underlying molecular mechanisms. The antitumor effect of AM was assessed on the subcutaneous tumors of human colorectal cancer cell line HCT116 grafted into nude mice. The mice were treated with either water or $500 \mathrm{mg} / \mathrm{kg}$ AM once per day, before being sacrificed for extraction of tumors, which were then subjected to microarray expression profiling. The gene expression of the extraction was then profiled using microarray analysis. The identified genes differentially expressed between treated mice and controls reveal that administration of AM suppresses chromosome organization, histone modification,
\end{abstract}

Correspondence to: Professor Li-Jen Su, Institute of Systems Biology and Bioinformatics, National Central University, 300 Jhongda Road, Jhongli, Taoyuan 320, Taiwan, R.O.C.

E-mail: sulijen@gmail.com

Professor Hoong-Chien Lee, Department of Physics, Chung Yuan Christian University, 200 Chung Pei Road, Jhongli, Taoyuan 320, Taiwan, R.O.C.

E-mail: hclee12345@gmail.com

Key words: colorectal cancer, microarray, microRNA, xenograft, Astragalus membranaceus and regulation of macromolecule metabolic process. A separate analysis focused on differentially expressed microRNAs revealing involvement of macromolecule metabolism, and intracellular transport, as well as several cancer signaling pathways. For validation, the input of the identified genes to The Library of Integrated Network-based Cellular Signatures led to many chemopreventive agents of natural origin that produce similar gene expression profiles to that of AM. The demonstrated effectiveness of AM suggests a potential therapeutic drug for colorectal cancer.

\section{Introduction}

Colorectal cancer (CRC) is the third most common cancer worldwide (1) and also the third leading cause of cancer-related death in Taiwan (2). The pathogenesis of colon cancer is unclear, but it is believed that family history, genetic alteration, diet and lifestyle changes are likely involved. The current cytotoxic drugs in the treatment of CRC include 5-fluorouracil (5-FU) alone or in combination with capecitabine, irinotecan and/or oxaliplatin (3). Although 5-FU has been used as the first-line treatment for colon cancer, the response rate is $<20 \%$ and patients who have responded may become resistant (4). In addition, serious side effects and complications (e.g., fatigue, pain, diarrhea, nausea, vomiting, and hair loss) can add extra burden to patients during treatment (5). Thus, it is necessary to seek a novel antitumor agent including phytochemical compounds in the treatment of CRC.

The dried root of Astragalus membranaceus (Fischer) Bge. var. mongolicus (Bge.) Hsiao (AM) is commonly used in the treatment of common cold, diarrhea, fatigue, and anorexia (6). The active constituents of AM include saponins, flavonoids and polysaccharides (7). Pharmacological studies on AM have demonstrated its effect on anti-oxidation, anti-inflammation 
and enhancement of immune system response (8). There are growing evidence that AM may be a potential anti-tumorigenic agent. For instance, AM was able to suppress hepatocarcinogenesis in rats (9). Formononetin, a type of isoflavonoid isolated from AM, presented anti-angiogenic effect in colon cancer cells in vitro and in vivo (10). Astragalus saponins have been studied extensively on tumor growth inhibition, decrease of cell invasiveness and angiogenesis, and induction of apoptosis (6,11-14). In combined therapy, 5-FU in combination with Astragalus polysaccharides enhances chemosensitivity of hepatoma cells (15). When in co-treatment with vinorelbine and cisplatin (VC) for patients with advanced non-small cell lung cancer, Astragalus polysaccharides have improved patients quality of life compared with VC alone (16).

Microarray technology provides a high throughput method to simultaneously analyze the gene expression levels of a given tissue or cell. It provides diagnostic and prognostic values in clinical trials because it allows investigators to identify the differentially expressed genes (DEGs) between samples. The differential labels are often referred to as biomarkers (17). Many common CRC genes have been characterized over the last decade. The three proposed classes of genes are oncogenes, tumor suppressor genes and stability genes (18). In addition to the expected frequently mutated genes (APC, TP53, $S M A D 4, P I K 3 C A$ and $K R A S$ ) found in CRC, other genes such as ARIDIA, SOX9 and FAM123B were also identified (19). Furthermore, the emerging role of microRNA (miRNA) in cancer chemoprevention was recently described (20-23). However, the changes in miRNA expression levels and how they are regulated by AM remains unclear.

Recently, the database Library of Integrated Networkbased Cellular Signatures (LINCS) was produced (24). It is an expression-based high-throughput screening application for repurposing biomedicines and for accelerating drug discovery. It works by comparing recorded profiles of gene expression induced by bioactive molecular perturbations to those the users had derived in their studies. The data was made available by the L1000 technology that generated approximately one million gene expression profiles from 22,412 unique perturbations applied to 56 different human primary cell lines and human cancer cell lines. LINCS does not only provide chemical perturbations, but also gives genetic perturbations such as knockdowns and overexpression of a single gene, a unique feature among similar applications.

In the present study, we investigated the antitumor effect of AM on human colorectal cancer HCT116 mouse xenograft and observed that AM can effectively reduce the tumor growth in nude mice. Using microarray analysis, we identified genes and miRNAs the expression levels and molecular mechanisms, and inferred that these changes may be associated with AM. Utilizing the LINCS database, we were able to identify chemopreventive drugs that have similar expression profile that represents the effect of AM. Our results suggested that AM, through gene regulation, could be a promising drug for cancer therapy.

\section{Materials and methods}

Ethics statement. All animal work was conducted according to relevant national and international guidelines. The Animal Use Protocol was reviewed and approved by the Institutional
Animal Care and Use Committee (IACUC) of Taichung Veterans General Hospital, Taiwan. The IACUC approval number is La-1021107. Period of protocol was valid from $04 / 01 / 2013$ to $03 / 31 / 2014$.

Cell culture condition. Human colorectal cancer cell line HCT116 was purchased from the American Type Culture Collection (ATCC; Manassas, VA, USA). The cancer cells were maintained in McCoy's 5A medium (Gibco, Carlsbad, CA, USA) supplemented with $10 \%$ fetal bovine serum, $100 \mathrm{U} / \mathrm{ml}$ penicillin and $100 \mathrm{mg} / \mathrm{ml}$ streptomycin (Biowest, Nuaille, France). Cells were cultured at $37^{\circ} \mathrm{C}$ in a humidified atmosphere of $5 \% \mathrm{CO}_{2}: 95 \%$ air.

Preparation of AM. The crude extract of AM was purchased from Sun Ten Pharmaceutical Corporation and identified by Brion Research Institute of Taiwan (batch no. M21051). The dried AM (100 g) was soaked in 1.51 water for $24 \mathrm{~h}$. The next day, the stock was decocted in $90^{\circ} \mathrm{C}$ distilled water for $6 \mathrm{~h}$. The decoction liquid was filtered and concentrated using a rotary evaporator, and lyophilized into powder. The dried powder was stored at $-20^{\circ} \mathrm{C}$ until use. The voucher specimen (accession no. BP002) is deposited in Professor Li-Jen Su laboratory, National Central University, Taiwan.

Tumor xenografts in nude mice. Three-week-old male

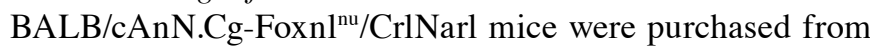
National Applied Research Laboratories (Taipei, Taiwan). All animals were housed in appropriate cages at $25^{\circ} \mathrm{C}$ on a 12-h light/dark cycle with access to food and water ad libitum. Animals were allowed a period of acclimatization before any experimentation. Tumors were established by subcutaneous injection of $1 \times 10^{6}$ HCT116 cells into the dorsal skin of mice using 25-G needles. Twelve days later, the animals were fed with $500 \mathrm{mg} / \mathrm{kg} \mathrm{AM}$ or only distilled water every day for 28 days ( $\mathrm{n}=8$ for each group). Tumor growth was monitored once a week for 4 weeks by measuring two perpendicular diameters. Tumor volume was calculated according to the formula: $\mathrm{a} \times \mathrm{b} \times(\mathrm{b} / 2)$, where $\mathrm{a}$ and $\mathrm{b}$ are the largest and smallest diameters, respectively. The eight p-values of tumor volume were calculated based on Benjamini-Hochberg method, which renders a false discovery rate of 0.1 . All animals were euthanized on day 40 and sacrificed by $\mathrm{CO}_{2}$ inhalation. The tumors were then collected for further analysis.

Microarray experiment. Total RNA was extracted using PureLink ${ }^{\circledast}$ RNA Mini kit (Ambion, Carlsbad, CA, USA) from cells treated with control or high doses of AM, according to the manufacturer's protocol. The concentration, purity and quality of total RNA for microarray analysis were determined using Nanodrop 2000 (Thermo Scientific, Wilmington, DE, USA) and had an $\mathrm{OD}_{260} / \mathrm{OD}_{280}$ ratio ranging from 1.9 to 2.1. The gene expression data was generated using Affymetrix Human Transcriptome Array 2.0 (HTA-2.0; Affymetrix, Santa Clara, CA, USA). The cDNA synthesis and labeling were carried out according to Affymetrix GeneChip ${ }^{\circledR}$ WT PLUS reagent protocol (Affymetrix). In brief, the total RNAs were first reverse transcribed to complementary DNA (cDNA) and then to complimentary RNAs (cRNAs) by in vitro transcription. The cRNAs were purified and quantified and the second cycle 
Table I. Primer sequences used in this study.

\begin{tabular}{llll}
\hline Gene & \multicolumn{1}{c}{ Forward } & \multicolumn{1}{c}{ Reverse } & \multicolumn{1}{c}{ Refseq } \\
\hline BRD2 & ATTCCAGCTCCTCCTCTTCC & GCAGAGCCAGCTCTCCTAGA & NM 001113182.2 \\
CREBBP & GGAAACCTTGAGCCATGTGT & CACAGGATGCAGACTCCAAA & NM 001079846.1 \\
HNRNPU & CAGAGCCAAATCTCCTCAGC & CTTTGCCTTTTGACACACCA & NM 004501 \\
KMT2D & CTCTGGATGGGATTGATGCT & CGTGGCTCTTCCTGTTCTC & NM 003482 \\
MYCBP2 & TCATCCATCCCTGTGTCTCA & AAAGAGGCAAGCACAGGAAA & NM 015057.4 \\
TRRAP & ACCTTGGTGTGTGGTGTCAA & GCCACACGGATGTATGTCTG & NM 003496 \\
\hline
\end{tabular}

single strand cDNA synthesis was performed using cRNAs as template. The RNA was removed and single strand cDNA was fragmented and hybridized to arrays. All the arrays were scanned with Affymetrix GeneChip 3000 7G (Affymetrix). The Affymetrix quality control report was produced to ensure the data quality of the processed arrays. All the arrays have passed the suggested values provided by Affymetrix and published on GEO (GEO accession no. GSE70772. http://www. ncbi.nlm.nih.gov/geo/query/acc.cgi?token=wnaxqakubzglvip $\&$ acc $=$ GSE70772).

Analysis of microarray gene expression profile. The genes differentially expressed in this study were selected using GeneSpring software, version GX 7.3 (Agilent, Santa Clara, CA, USA). The $\log _{2}$-transformed expression intensities with Robust Multiarray Average (RMA) normalization and Principle Component Analysis (PCA) from 16 arrays $(n=8$ in each group) were performed. According to PCA, a total of only 8 arrays were used ( $n=4$ in each group) to calculate the correlation coefficient in each cluster set and heatmap was constructed. The t-test $\mathrm{p}$-value and fold-change for comparing the differentially gene expression in the treatment and control group were calculated. A p-value $<0.05$ and a fold-change of $>1.5$ were considered as differentially expressed. The Fisher's exact test was applied to identify overrepresented Gene Ontology (GO) terms (25) (http://geneontology.org). A $\mathrm{P}<0.001$ was considered statistically significant.

Real-time PCR validation. For each sample, $2.5 \mu \mathrm{g}$ of RNA was converted into cDNA using SuperScript ${ }^{\circledR}$ VILO $^{\text {TM }}$ MasterMix (Invitrogen, Carlsbad, CA, USA) according to manufacturer's protocol. All primer sets were designed using NCBI PrimerBLAST and each primer pair was checked for primer-dimer using Beacon designer software version 8.13 from Premier Biosoft International (Palo Alto, CA, USA). The primers were further checked using melt curve analysis. The reaction was performed on ABI ViiA ${ }^{\mathrm{TM}} 7$ Real-Time PCR system (Applied Biosystems, Life Technologies, Foster City, CA, USA) using RealQ Plus 2X Master Mix Green as dye (Ampliqon, Odense, Denmark) according to manufacturer's manual. The reaction condition is as followed: $95^{\circ} \mathrm{C} 15 \mathrm{~min}$ followed by $\left(98^{\circ} \mathrm{C}\right.$ : $15 \mathrm{sec}, 60^{\circ} \mathrm{C}$ : $\left.30 \mathrm{sec}, 72^{\circ} \mathrm{C}: 30 \mathrm{sec}\right) \mathrm{x} 40$ cycles. All reactions were carried out with four biological replicates, and each analysis consists of two technical replicates. The signal of housekeeping gene GAPDH was used for normalization and the relative expression levels were calculated using the $2^{-\Delta \Delta \mathrm{Ct}}$ method. The primer sequences are shown in Table I.
Database analysis for differentially expressed miRNAs. Cytoscape was utilized to construct the miRNA-target gene network (26). The target genes of miRNAs were identified from miRTarBase (http://mirtarbase.mbc.nctu.edu.tw) (27) and were subjected to DAVID for GO biological process analysis. The selected terms were based on Benjamini corrected $\mathrm{P}<0.05$. miRNA pathway analysis was predicted using DIANA-miRPath (http://diana.imis.athena-innovation. gr/DianaTools/index.php?r=mirpath/index) (28) with FDR corrected $\mathrm{P}<0.05$.

Identification of compound signature using LINCS. LINCS or the Library or Integrated Network-based Cellular Signatures project is the extensive version of CMAP (29) which is available on the http://lincscloud.org website. Detail of the website can be found as published (24). In brief, the up and downregulated gene lists are inserted to the textbox on the search interface. Click on the search button will reveal lists of matching experiments in a table format. The three tables are 'compound connection', 'consensus knockdown connection' and 'overexpression connection'. For this study, we only focus on compound connection. The compound selection criteria were based on the mean connectivity score across four cell lines with a magnitude between -90 and -99 , which corresponds to significant reverse connections.

Statistical analysis. Statistical analysis was performed using Student's t-test. Differences were considered statistically significant with $\mathrm{P}<0.05$ unless otherwise specified.

\section{Results}

The inhibitive effects of AM in vivo. To test the effect of AM on tumor growth inhibition in vivo, a human colorectal cancer xenograft nude mouse model was generated. The mice were orally fed with water or $500 \mathrm{mg} / \mathrm{kg}$ AM for 28 days. On the final day, mice were sacrificed and tumors were inoculated for further studies. As shown in Fig. 1A and B, the tumor volume and final tumor weight of $500 \mathrm{mg} / \mathrm{kg}$ AM groups were reduced by $47.5 \%\left(380 \pm 109 \mathrm{~mm}^{3}\right.$ in control vs. $199 \pm 69 \mathrm{~mm}^{3}$ in AST-treated mice after 28 days) and $48.4 \%(2.56 \pm 0.49 \mathrm{~g}$ in control vs. $1.32 \pm 0.74 \mathrm{~g}$ in AST-treated mice after 28 days) compared to control, respectively. However, in no group throughout the experiment, was significant changes in body weight observed (Fig. 1C), or mortality recorded, unlike the common side effects of traditional chemotherapy. In addition, the non-tumor-bearing mouse groups fed with $500 \mathrm{mg} / \mathrm{kg} \mathrm{AM}$ 

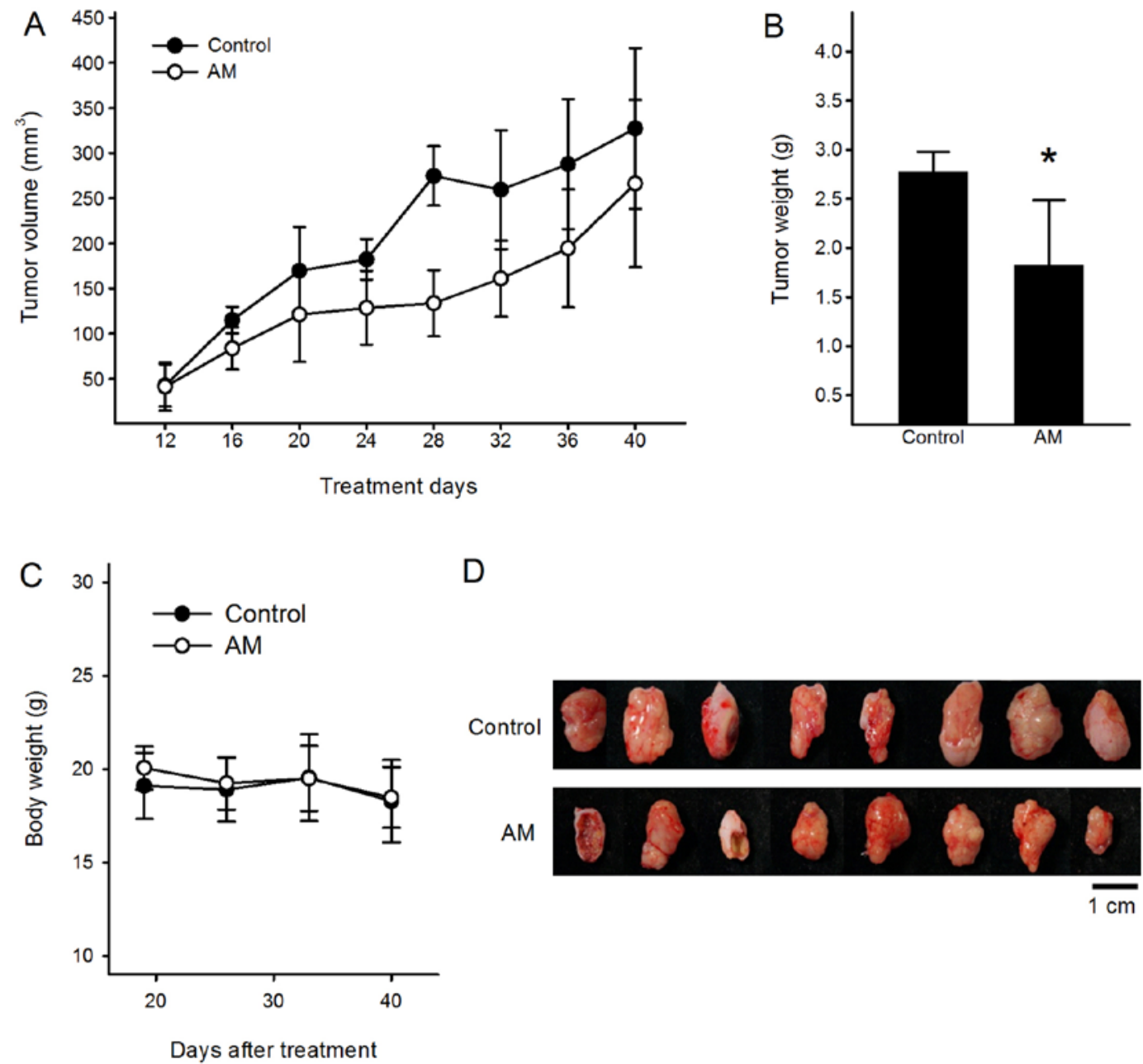

Figure 1. Administration of AM inhibits tumor growth of HCT116-induced mouse xenografts. The mice were treated orally every day with either control or $500 \mathrm{mg} / \mathrm{kg} \mathrm{AM}$ after the tumors became palpable at the 12th day. Effects of AM on (A) tumor volume, (B) final tumor weight and (C) body weight were measured. (D) Pictures showing the excised tumors on the 40th day from the two groups. The means and variances were evaluated based on $\mathrm{n}=8 \mathrm{mice}$ per group. ${ }^{*} \mathrm{P}<0.05$ compared to control.

did not show any toxicity or change in body weight during the course of experiment (data not shown). Next, the excised tumors were evaluated for microarray analysis (Fig. 1D).

Bioinformatics analysis of molecular mechanisms. The tumors were processed for microarray investigation to determine the differential transcriptional profiles between control and AM-treated groups. After normalization of the microarray data, a total of 1,454 DEGs were identified, including 1,257 upregulated and 197 downregulated genes at the threshold of $\mathrm{P}<0.05$ and fold-change $>1.5$. The heatmap of gene expression patterns between control and treatment groups is shown in Fig. 2A. The significant changes between control and treatment groups warrant further study.

The DEGs were mapped onto GO for cellular component, molecular function and biological process analyses. A total of 182 enriched terms covering 224 genes were in the upregulated GO categories. In Fig. 2B, 6 out of 38 terms were listed under cellular component category. The listed terms include 'intracellular part', 'organelle part', 'organelle', 'macromolecular complex', 'cell junction' and 'cell leading edge'. In the molecular function category, 7 out of 45 terms were listed. The enriched terms include 'heterocyclic compound binding', 'organic cyclic compound binding', 'small molecule binding', 'chromatin binding', 'hydrolase activity, acting on acid anhydrides', 'cytoskeletal protein binding' and 'carbohydrate derivative binding'. In the biological process category, a total of 26 out of 99 enriched terms were listed. AM has been widely studied for its capability of immunopotentiation (30), apoptosis induction $(6,31)$ and inhibition of inflammation (32) and invasion (12). The possibilities that AM may function through suppressing other emerging cancer hallmarks, such as genome destabilization and cellular energetics deregulation, remain rarely studied. To understand such possibilities, we focus the following discussion on three of the enriched biological processes, which are 'chromosome organization' (36 genes, $\mathrm{P}<0.001)$, 'histone modification' $(13$ genes, $\mathrm{P}<0.001)$ and 'regulation of macromolecule metabolic process' (86 genes, $\mathrm{P}<0.001)$. More specifically at the gene level, we selected six of the DEGs to quantitatively demonstrate the effects of AM on their expression levels. The selected genes are bromodomain containing 2 (BRD2), CREB binding protein (CREBBP), heterogeneous nuclear ribonucleoprotein U (HNRNPU), lysine (K)-specific methyltransferase 2D (KMT2D), MYC binding protein 2, E3 ubiquitin protein ligase (MYCBP2), and transformation/transcription domain-associated protein 
A
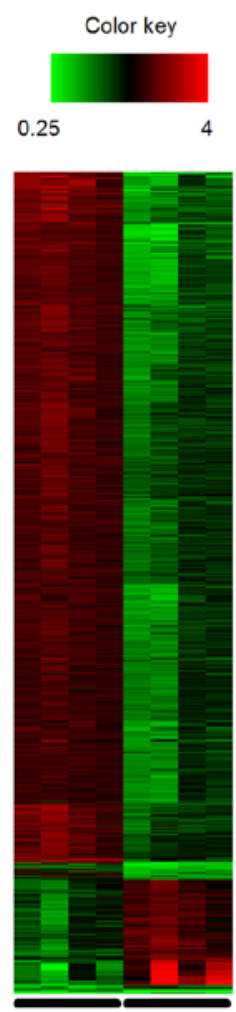

Control AM
B

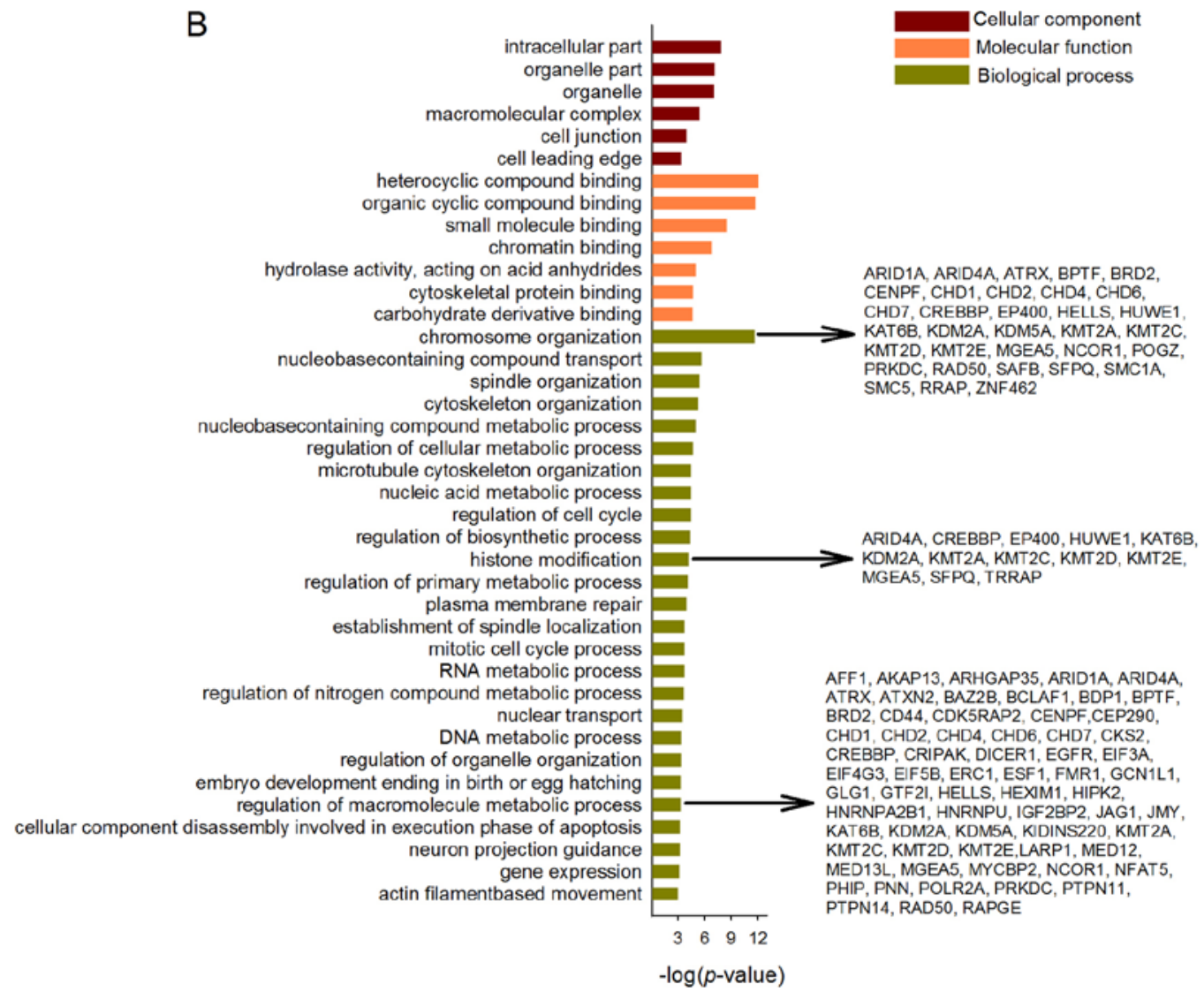

Figure 2. Bioinformatics analysis of HCT116-induced mouse xenografts treated with AM. (A) Heatmap comparison of the 1,454 DEGs between controls and AM-treated group. Each column shows a profile of a specimen across genes, while each row shows a profile of a gene across specimens. Red color indicates upregulation and green color indicates downregulation. (B) GO analysis showing cellular components, molecular functions and biological processes enriched by the 224 genes. The corresponding genes of the biological processes of interest are indicated by arrows.
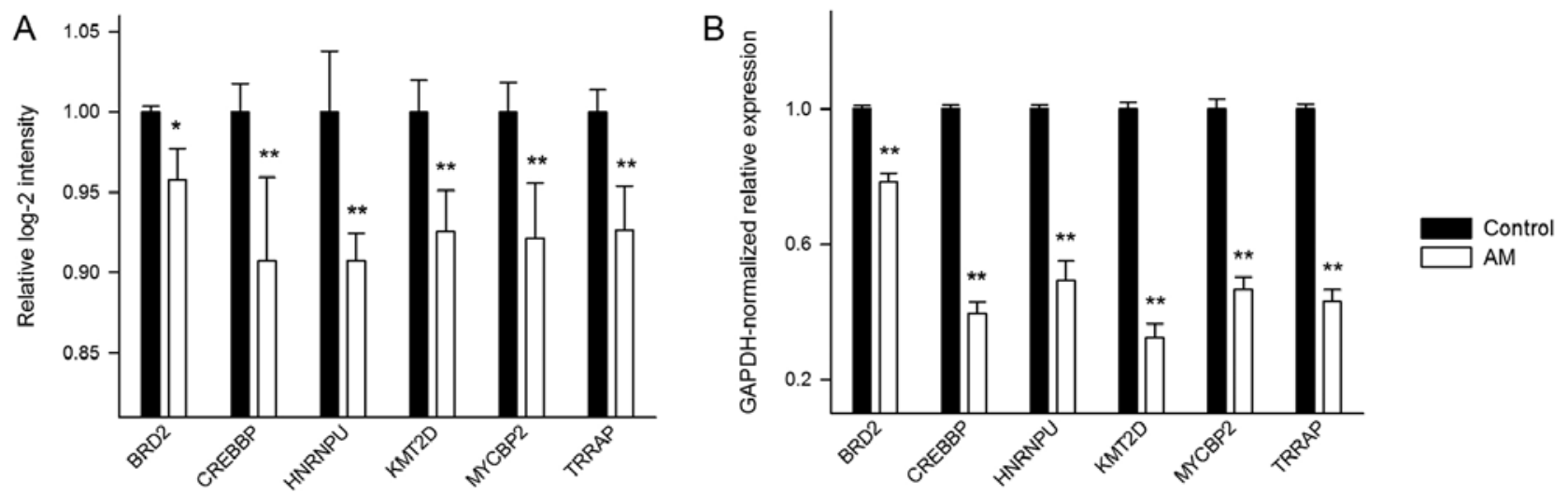

Figure 3. Pattern consistency of AM-induced expression changes between the microarray analysis and the real-time PCR analysis. (A) Microarray expression levels of the six selected genes in the AM-treated group and the control group. (B) Real-time PCR-rendered expression changes showing a similar pattern to the microarray results. ${ }^{*} \mathrm{P}<0.05$ and ${ }^{* *} \mathrm{P}<0.01$ compared to control.

(TRRAP) (Fig. 3A). All the six genes were downregulated under AM treatment. The respective downregulated and upregulated DEGs in the control and treatment groups did not enrich any GO category.

To confirm the above microarray results, we performed real-time PCR to quantify the expression changes of the six selected genes (Fig. 3B). The results show that the genes have wide ranges of $\mathrm{Ct}$ values from 20.54 to 33.72 . Overall, the real-time PCR expression changes exhibit a consistent pattern with that from the microarray analysis.

Focal analysis on miRNA. Interestingly, the Affymetrix HTA 2.0 array covers $>40,000$ non-coding transcripts on the chip and a total of 29 miRNAs were expressed among the 1,425 DEGs presented on the heatmap. To eludicate the functions of the miRNAs, miRTarBase was utilized to identify 
A

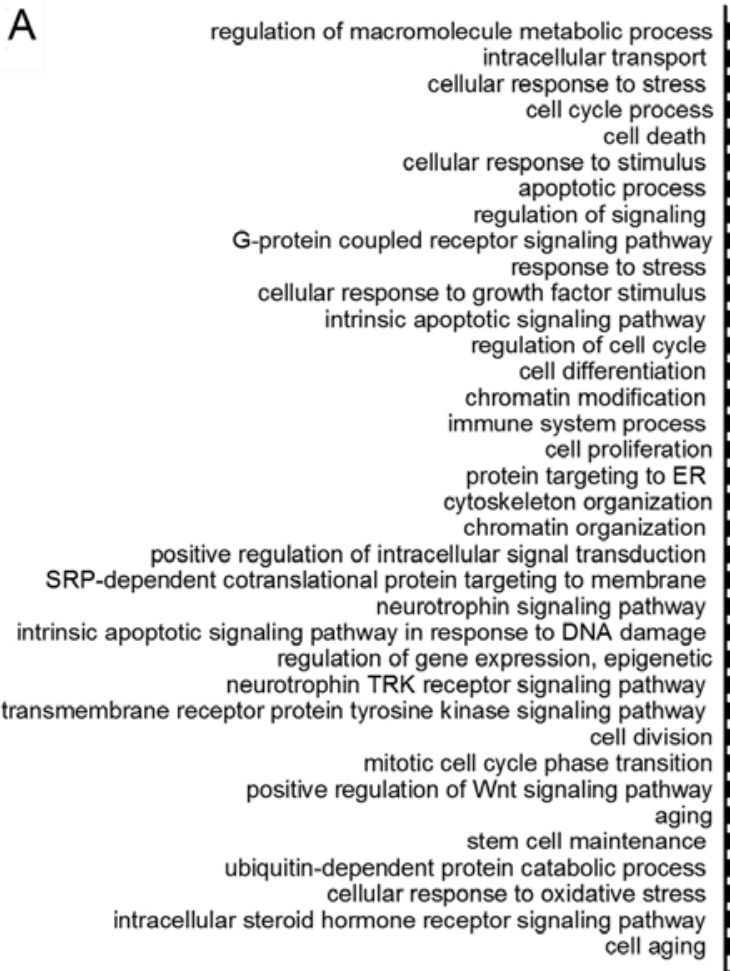

$$
\begin{gathered}
1020 \\
-\log (p \text {-value })
\end{gathered}
$$

C
B
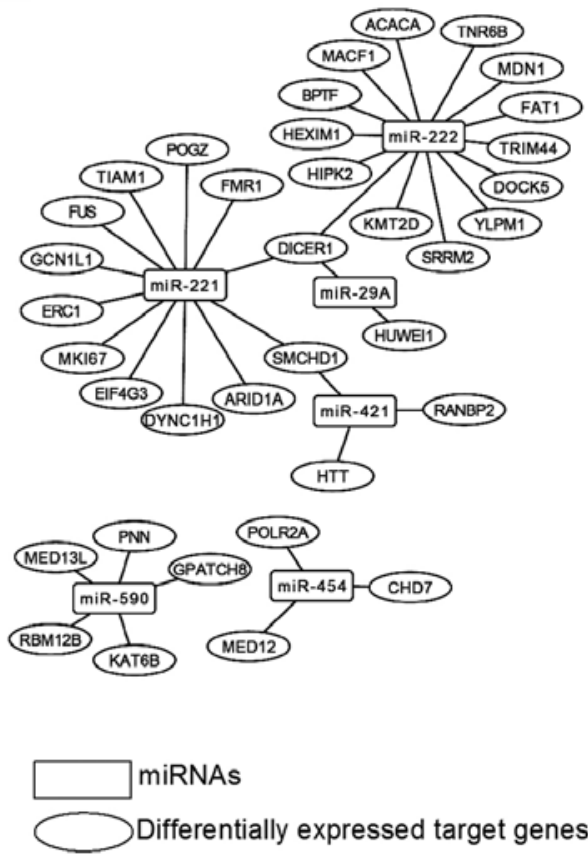

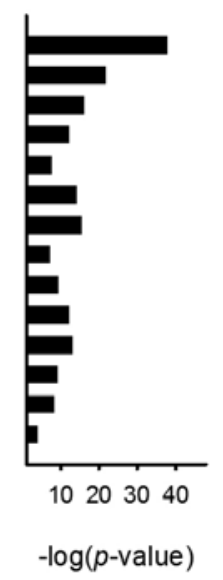

Figure 4. Differentially expressed miRNAs and their target genes. (A) Biological processes enriched by the 885 miRTarBase listed target genes. (B) Interaction network of the 37 differentially expressed miRNA target genes and their targeting miRNAs. Rectangles are miRNAs and ovals are target genes. Both the miRNAs and the differentially expressed target genes were found upregulated. (C) The 14 cancer related pathways obtained using the DIANA-miRPath database and the 29 differentially expressed miRNAs as input.

the target genes. A total of 885 target genes were identified including 37 DEGs. GO enrichment biological process analysis of the target genes was associated with terms such as 'regulation of macromolecule metabolic process', 'intracellular transport', 'cellular response to stress', 'cell cycle process', 'cell death', 'regulation of signaling, 'chromatin modification', 'immune system process', 'cell proliferation', 'transmembrane receptor protein tyrosine kinase signaling pathway' and 'positive regulation of Wnt signaling pathway' (Fig. 4A). The network of miRNAs and differentially expressed target genes are shown in Fig. 4B. These genes were tumor suppressorrelated (ARIDIA, FAT1, HIPK2, MDN1, MED13L, RANBP2, $P N N$, and $S M C H D I)$, invasion and migration-related (BPTF,
DOCK5, MED12, TIAMI and TRIM44), and cell proliferationrelated (BPTF, DICER1, FUS, HEXIM1, KAT6B, MKI67, and POLR2A). The pathway analysis of miRNA predicted target genes were analyzed using DIANA-miRPath. A total of 100 biological pathways were identified, including many wellknown cancer-related pathways such as PI3k/Akt signaling pathway, MAPK signaling pathway, HIF-1 signaling pathway, JAK/STAT signaling pathway and VEGF signaling pathway. PI3K/Akt pathway has the most identified target genes among the predicted biological pathways and miR-590 was present in all of the selected 14 pathways (Fig. 4C). Around $40 \%$ of the pathways are implicated in carcinogenesis, suggesting the novel role of AM on miRNA regulation. 
Table II. The top perturbagen hits from LINCS.

\begin{tabular}{llcl}
\hline Perturbagen ID & Perturbagen name & Score & Therapeutic use \\
\hline BRD-A02481876 & Importazole & -100.0 & None \\
BRD-A36630025 & SN-38 & -99.9 & Anti-neoplastic \\
BRD-A48237631 & Mitomycin-c & -99.7 & Antibiotic \\
BRD-A80960055 & Celastrol & -99.6 & Anti-inflammation \\
BRD-A35588707 & Teniposide & -99.4 & Anti-neoplastic \\
BRD-K98490050 & Amsacrine & -99.4 & Anti-neoplastic \\
BRD-A59985574 & Topotecan & -99.3 & Anti-neoplastic \\
BRD-A30437061 & Camptothecin & -99.2 & Anti-neoplastic \\
BRD-K08547377 & Irinotecan & -98.5 & Anti-neoplastic \\
BRD-K31542390 & Mycophenolic-acid & -98.4 & Immunosuppressant \\
BRD-K80622725 & STK-397047 & -97.8 & None \\
BRD-K31342827 & Bisindolylmaleimide & -97.6 & None \\
BRD-A18419789 & Etoposide & -97.4 & Anti-neoplastic \\
BRD-K53792571 & Inhibitor-BEC & -97.4 & None
\end{tabular}

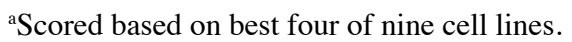

Drug predictions using LINCS. LINCS derives perturbagents of both aggravate or reverse directions, thus allowing users to identify lists of harmful or beneficial drugs for possible therapeutic treatments. To search for small molecules that have similar effect as AM, that it, decrease in gene expression after administration of drug, we have identified a total of 71 significant perturbagens. The top 14 perturbagens are listed in Table II. The drugs include antitumor effect such as SN-38, teniposide, amsarcrine, topotecan, camptothecin, irinotecan and etoposide. Other therapeutic drugs such as mitomycin-c, celastrol and mycophenolic acid were also listed. The results suggested that the effect of AM is similar to the perturbagens listed in Table II. This provides support for the reliability of our microarray study.

\section{Discussion}

In the present study, we have demonstrated that AM can effectively reduce the tumor growth in nude mice without significantly altering the mouse body weight. DEGs extracted from microarray gene expression profile indicate statistically significant functional changes including catalytic activity, binding, cellular component, biological process, biological regulation and cellular component organization. These changes correlate well with morphological changes in mouse tumors. For example, terms such as chromosome organization, histone modification and regulation of macromolecule metabolic process were upregulated and downregulated in control and treatment groups, respectively. These terms are rarely described in TCM, suggesting a new therapeutic use of AM.

Our microarray data revealed that many epigenetic-related genes including $K M T 2 D, B R D 2, C R E B B P, A R I D 1 A$, were altered in AM-treated groups. Hsieh et al tested 3,294 TCMs and found that $29.8 \%$ have the potential to affect the epigenome of the human cells (33). Epigenetics is the change in gene expression independent of alteration in DNA sequences (34).
Its modification involves changes in DNA methylation, histone modification and miRNAs expression (23). In cancer cells, two forms of epigenetics are commonly observed. One is hypomethylation of the proto-oncogenes or increase in expression of genes implicated in tumor progression. On the other hand, tumor suppressor genes were silenced due to hypermethylation on their promoter region. These reactions are catalyzed by a family of enzymes called DNA methyltransferases (DNMTs), histone acetyltransferase (HATs) and histone deacetylase (HDACs) $(35,36)$. KMT2D is an important component of the multi-protein complex that directs epigenetics regulated genes and embryonic development $(37,38)$. KMT $2 D$ are frequently mutated in malignant cells. For instance, $K M T 2 D$ contains recurrent mutation in $90 \%$ of the cases in follicular lymphoma (39). Furthermore, high levels of KMT2D are associated with breast and colon cancer malignancies (40). Another property of $K M T 2 D$ is its ability to promote cell migration (41); thus it is possible that the reduction of $K M T 2 D$ in the treatment group was affected by AM since the roots of Astragalus membranaceus and/or Astragalus saponins have been shown to attenuate invasion and migration of cancer cell lines $(12,42)$. Interestingly, $K M T 2 D$ also plays a part in regulating calcium signaling pathways $(41,43)$ and deregulation of this pathway may mediate tumor growth (44-46). Astragaloside IV, one of the main constituents found in AM, was able to reduce the excess intracellular calcium by improving the activity of calcium pump in myocardial cells $(8,47)$, suggesting astragaloside IV is valuable in enhancing tumor inhibition by implicating the calcium signaling pathway.

Small molecules targeting bromodomain, especially the BET family ( $B R D 1, B R D 2, B R D 3, B R D 4$, and $B R D T)$, can be a promising therapeutic strategy in cancer treatment. The bromodomain is an evolutionarily conserved protein that act as 'reader' of histone acetylation and regulates protein-protein interaction via lysine residue $(34,48)$. BRD2, the potential oncogene in humans, was downregulated in our mouse xenograft 
treatment group. A few BET protein inhibitors were designed to target the acetylated histones and the bromodomains (49) and inhibition of BET proteins can ultimately reduce the transcription of proto-oncogenes such as MYC, BCL2 and CDK6 (50). Furthermore, $B R D 2$ expression can result in B cell maglinancy, which is activated by nuclear factor- $\kappa \mathrm{B}(\mathrm{NF}-\kappa \mathrm{B})$-regulated genes (51). Consistent with this finding, another study has shown that $B R D 2$ can directly regulate $N F-\kappa B$ activity in a macrophage cell line and downregulation of $B R D 2$ ultimately decreases NF- $\kappa \mathrm{B}$ activity (52). NF- $\kappa \mathrm{B}$ is pivatol in the control of inflammation, cell proliferation and apoptosis. AM has long been known for its anticancer effect by activating anti-inflammatory and immune boost properties. For instance, saponins found in AM were able to induce tumor growth inhibition and promote apoptosis through ERK-independent NF- $\kappa \mathrm{B}$ signaling pathway (11). We suspected that $\mathrm{AM}$ regulates $\mathrm{NF}-\kappa \mathrm{B}$ activity by altering $B R D 2$ expression.

Energy metabolism reprogramming is one of the emerging hallmarks of many cancers $(53,54)$. One of the factors facilitating uncontrollable proliferation of cancer cells is their high rates of aerobic glycolysis, a phenomenon known as the 'Warburg effect' $(55,56)$. The factor affords tumor cells even more abundant ATPs than can be produced through oxidative phosphorylation. The degraded glucose through glycolysis recursively fuels proliferation by providing intermediates needed for synthesizing amino acids and nucleosides $(53,57)$, the respective building blocks of protein and DNA. MYC is one of the main oncogenes in cancer cells by promoting glucose transporter 1 (GLUT1) and contributes to the Warburg effect (54). In our microarray profile, we have identified $M Y C$-associated genes such as MYCBP2 (58), HNRNPU (59) and TRRAP (60), all of which play a role in regulating $M Y C$ expression, as having been downregulated by AM. Studies have shown that $M Y C B P 2$ positively regulates with mTOR signaling (61), which controls the metabolic reprogramming of cancer cells (62), and during metabolic stress, mTOR is inactivated by AMP-activated protein kinase (AMPK) (54). Astragalus polysacchride is known to activate AMPK to alleviate glucose toxicity in diabetes (63). Furthermore, drugs such as metaformin and phenformin that were developed to target type 2 diabetes may have use in treating cancers by triggering AMPK in cells $(64,65)$, suggesting AM, like metaformin or phenformin, is an excellent adjuvant drug possibly by activating AMPK and ultimately decreasing mTOR and MYCBP2 expression.

miRNAs are a family of small, evolutionary conserved, endogenous and non-coding RNAs that regulate gene expression in broad range of animals, plants and viruses. They play critical regulatory roles in a large number of biological processes such as cellular differentiation, proliferation, migration and apoptosis $(66,67)$. Several studies had focused on TCMs and their effect on miRNA expression. For example, curcumin and its derivatives were able to alter miRNA signature profiles in various cancers including colon $(23,68)$. Bufalin, one of the active ingredients of Venenum bufonis, can inhibit colon cancer metastasis by acting synergistically with miR-497 (69). However, studies on AM in modulating miRNA expression in cancers are limited. Here, we identified 29 differentially expressed miRNAs and found that miR-590 has the most identified target genes and is involved in all of the selected
miRNA predictive pathways. miR-590, a possible oncomiR, was overexpressed in many tumors (70-72). Database mining identified TGFBR2 is one of the target genes of miR-590-5p. Studies have found that mutation of TGFBR2 occurs in $25 \%$ of CRC and in $>90 \%$ of microsatellite instability-high CRC (73). These findings shed light on the additional roles of AM in miRNA regulation.

The results from LINCS indicate that many chempreventive drugs have profiles similar to that of AM. Interestingly, many of the identified antitumor agents were of natural origin. For example, etoposide and teniposide are the derivative of podophyllotoxin which was found in the plant Podophyllum peltatum, SN-38, topotecan and irinotecan are the derivatives of camptothecin which was isolated from Camptotheca acuminata (74). Celastrol is another TCM-isolated chemical compound from the root of Trypterigium wilfordii. More recently, studies have shown that celastrol, in addition to its anti-inflammatory activities, can inhibit proliferation of various human cancer cells (75). We have also identified curcumin (score: -92.5) as another natural product that have similar gene expression effect as AM. Curcumin is by far the most studied TCM-based anticancer drug. It can modulate the growth of tumor cells by regulating several pathways such as NF- $\mathrm{B}, \mathrm{PI} 3 \mathrm{~K} / \mathrm{Akt}$, JAK/STAT, AMPK and mTOR, all of which were also observed in AM.

Although more work is needed to further clarify the effects of AM at the molecular level, our preliminary results show that crude extract of AM inhibits growth of CRC in vivo without apparent toxicity and side effect. Our bioinformatics analysis provides preliminary insight into the molecular mechanisms of AM, while the identified genes and miRNAs offer clues to explaining the reduced growth of mouse xenografted tumors. Our derived gene expression profile provides valuable information for selecting biomarkers and could contribute to future discovery of novel drugs for CRC.

\section{Acknowledgements}

The present study was supported by grants from the Ministry of Science and Technology (NSC 101-2320-B-008-001-MY3 and MOST-104-2320-B-182A-010). AM is kindly provided by Sun Ten Pharmaceutical Corporation. The authors thank Professor Sun-Chong Wang from National Central University for valuable comments and technical support from the Core Facility of High Throughput Experimental Analysis from Institute of Systems Biology and Bioinformatics, National Central University, Taiwan. The Core Facility of High Throughput Experimental Analysis is supported by Aim for Top University Project from the Ministry of Education.

\section{References}

1. Ferlay J, Shin HR, Bray F, Forman D, Mathers C and Parkin DM: Estimates of worldwide burden of cancer in 2008: GLOBOCAN 2008. Int J Cancer 127: 2893-2917, 2010.

2. Su SY, Huang JY, Jian ZH, Ho CC, Lung CC and Liaw YP: Mortality of colorectal cancer in Taiwan, 1971-2010: Temporal changes and age-period-cohort analysis. Int J Colorectal Dis 27: 1665-1672, 2012.

3. Dietvorst $\mathrm{MH}$ and Eskens FA: Current and novel treatment options for metastatic colorectal cancer: Emphasis on Aflibercept. Biol Ther 3: 25-33, 2013. 
4. Wong CS, Wong VW, Chan CM, Ma BB, Hui EP, Wong MC, Lam MY, Au TC, Chan WH, Cheuk W, et al: Identification of 5-fluorouracil response proteins in colorectal carcinoma cell line SW480 by two-dimensional electrophoresis and MALDI-TOF mass spectrometry. Oncol Rep 20: 89-98, 2008.

5. Qi F, Li A, Inagaki Y, Gao J, Li J, Kokudo N, Li XK and Tang W: Chinese herbal medicines as adjuvant treatment during chemo- or radio-therapy for cancer. Biosci Trends 4: 297-307, 2010.

6. Tin MM, Cho CH, Chan K, James AE and Ko JK: Astragalus saponins induce growth inhibition and apoptosis in human colon cancer cells and tumor xenograft. Carcinogenesis 28: 1347-1355, 2007.

7. Ma XQ, Shi Q, Duan JA, Dong TT and Tsim KW: Chemical analysis of Radix Astragali (Huangqi) in China: A comparison with its adulterants and seasonal variations. J Agric Food Chem 50: 4861-4866, 2002.

8. Ren S, Zhang H, Mu Y, Sun M and Liu P: Pharmacological effects of Astragaloside IV: A literature review. J Tradit Chin Med 33: 413-416, 2013

9. Cui R, He J, Wang B, Zhang F, Chen G, Yin S and Shen H: Suppressive effect of Astragalus membranaceus Bunge on chemical hepatocarcinogenesis in rats. Cancer Chemother Pharmacol 51: 75-80, 2003.

10. Auyeung KK, Law PC and Ko JK: Novel anti-angiogenic effects of formononetin in human colon cancer cells and tumor xenograft. Oncol Rep 28: 2188-2194, 2012.

11. Auyeung KK, Law PC and Ko JK: Astragalus saponins induce apoptosis via an ERK-independent $\mathrm{NF}-\kappa \mathrm{B}$ signaling pathway in the human hepatocellular HepG2 cell line. Int J Mol Med 23: 189-196, 2009.

12. Auyeung KK, Woo PK, Law PC and Ko JK: Astragalus saponins modulate cell invasiveness and angiogenesis in human gastric adenocarcinoma cells. J Ethnopharmacol 141: 635-641, 2012

13. Law PC, Auyeung KK, Chan LY and Ko JK: Astragalus saponins downregulate vascular endothelial growth factor under cobalt chloride-stimulated hypoxia in colon cancer cells. BMC Complement Altern Med 12: 160, 2012

14. Wang T, Xuan X, Li M, Gao P, Zheng Y, Zang W and Zhao G: Astragalus saponins affect proliferation, invasion and apoptosis of gastric cancer BGC-823 cells. Diagn Pathol 8: 179, 2013.

15. Tian QE, De Li H, Yan M, Cai HL, Tan QY and Zhang WY: Effects of Astragalus polysaccharides on P-glycoprotein efflux pump function and protein expression in $\mathrm{H} 22$ hepatoma cells in vitro. BMC Complement Altern Med 12: 94, 2012.

16. Guo L, Bai SP, Zhao L and Wang XH: Astragalus polysaccharide injection integrated with vinorelbine and cisplatin for patients with advanced non-small cell lung cancer: Effects on quality of life and survival. Med Oncol 29: 1656-1662, 2012

17. Chu W, Ghahramani Z, Falciani F and Wild DL: Biomarker discovery in microarray gene expression data with Gaussian processes. Bioinformatics 21: 3385-3393, 2005

18. Vogelstein B and Kinzler KW: Cancer genes and the pathways they control. Nat Med 10: 789-799, 2004.

19. Cancer Genome Atlas Network: Comprehensive molecular characterization of human colon and rectal cancer. Nature 487: 330-337, 2012

20. Karius T, Schnekenburger $M$, Dicato $M$ and Diederich $M$ : MicroRNAs in cancer management and their modulation by dietary agents. Biochem Pharmacol 83: 1591-1601, 2012.

21. Li Y, Kong D, Wang Z and Sarkar FH: Regulation of microRNAs by natural agents: An emerging field in chemoprevention and chemotherapy research. Pharm Res 27: 1027-1041, 2010.

22. Neelakandan K, Babu P and Nair S: Emerging roles for modulation of microRNA signatures in cancer chemoprevention. Curr Cancer Drug Targets 12: 716-740, 2012.

23. Teiten MH, Dicato M and Diederich M: Curcumin as a regulator of epigenetic events. Mol Nutr Food Res 57: 1619-1629, 2013

24. Duan Q,Flynn C, Niepel M, Hafner M, Muhlich JL, Fernandez NF Rouillard AD, Tan CM, Chen EY, Golub TR, et al: LINCS Canvas Browser: Interactive web app to query, browse and interrogate LINCS L1000 gene expression signatures. Nucleic Acids Res 42: W449-W460, 2014.

25. Ashburner M, Ball CA, Blake JA, Botstein D, Butler $\mathrm{H}$ Cherry JM, Davis AP, Dolinski K, Dwight SS, Eppig JT, et al: Gene ontology: Tool for the unification of biology. The Gene Ontology Consortium. Nat Genet 25: 25-29, 2000.

26. Smoot ME, Ono K, Ruscheinski J, Wang PL and Ideker T: Cytoscape 2.8: New features for data integration and network visualization. Bioinformatics 27: 431-432, 2011.
27. Hsu SD, Tseng YT, Shrestha S, Lin YL, Khaleel A, Chou CH, Chu CF, Huang HY, Lin CM, Ho SY, et al: miRTarBase update 2014: An information resource for experimentally validated miRNA-target interactions. Nucleic Acids Res 42: D78-D85, 2014.

28. Vlachos IS, Kostoulas N, Vergoulis T, Georgakilas G, Reczko M, Maragkakis M, Paraskevopoulou MD, Prionidis K, Dalamagas T and Hatzigeorgiou AG: DIANA miRPath v.2.0: Investigating the combinatorial effect of microRNAs in pathways. Nucleic Acids Res 40: W498-W504, 2012.

29. Lamb J: The Connectivity Map: A new tool for biomedical research. Nat Rev Cancer 7: 54-60, 2007.

30. Shao BM, Xu W, Dai H, Tu P, Li Z and Gao XM: A study on the immune receptors for polysaccharides from the roots of Astragalus membranaceus, a Chinese medicinal herb. Biochem Biophys Res Commun 320: 1103-1111, 2004.

31. Auyeung KK, Mok NL, Wong CM, Cho CH and Ko JK: Astragalus saponins modulate $\mathrm{mTOR}$ and ERK signaling to promote apoptosis through the extrinsic pathway in HT-29 colon cancer cells. Int J Mol Med 26: 341-349, 2010.

32. Qin Q, Niu J, Wang Z, Xu W, Qiao Z and Gu Y: Astragalus membranaceus inhibits inflammation via phospho-P38 mitogen-activated protein kinase (MAPK) and nuclear factor (NF)- $\mathrm{KB}$ pathways in advanced glycation end product-stimulated macrophages. Int J Mol Sci 13: 8379-8387, 2012.

33. Hsieh HY, Chiu PH and Wang SC: Epigenetics in traditional chinese pharmacy: A bioinformatic study at pharmacopoeia scale. Evid Based Complement Alternat Med 2011: 816714, 2011.

34. Dawson MA and Kouzarides T: Cancer epigenetics: From mechanism to therapy. Cell 150: 12-27, 2012.

35. Virani S, Colacino JA, Kim JH and Rozek LS: Cancer epigenetics: A brief review. ILAR J 53: 359-369, 2012.

36. Feinberg AP and Tycko B: The history of cancer epigenetics. Nat Rev Cancer 4: 143-153, 2004.

37. Demers C, Chaturvedi CP, Ranish JA, Juban G, Lai P, Morle F, Aebersold R, Dilworth FJ, Groudine M and Brand M: Activator-mediated recruitment of the MLL2 methyltransferase complex to the beta-globin locus. Mol Cell 27: 573-584, 2007.

38. Glaser S, Lubitz S, Loveland KL, Ohbo K, Robb L, Schwenk F, Seibler J, Roellig D, Kranz A, Anastassiadis K, et al: The histone 3 lysine 4 methyltransferase, Mll2, is only required briefly in development and spermatogenesis. Epigenetics Chromatin 2: $5,2009$.

39. Morin RD, Mendez-Lago M, Mungall AJ, Goya R, Mungall KL, Corbett RD, Johnson NA, Severson TM, Chiu R, Field M, et al: Frequent mutation of histone-modifying genes in non-Hodgkin lymphoma. Nature 476: 298-303, 2011.

40. Natarajan TG, Kallakury BV, Sheehan CE, Bartlett MB, Ganesan N, Preet A, Ross JS and Fitzgerald KT: Epigenetic regulator MLL2 shows altered expression in cancer cell lines and tumors from human breast and colon. Cancer Cell Int 10: $13,2010$.

41. Guo C, Chen LH, Huang Y, Chang CC, Wang P, Pirozzi CJ, Qin X, Bao X, Greer PK, McLendon RE, et al: KMT2D maintains neoplastic cell proliferation and global histone $\mathrm{H} 3$ lysine 4 monomethylation. Oncotarget 4: 2144-2153, 2013.

42. Wu JJ, Sun WY, Hu SS, Zhang S and Wei W: A standardized extract from Paeonia lactiflora and Astragalus membranaceus induces apoptosis and inhibits the proliferation, migration and invasion of human hepatoma cell lines. Int J Oncol 43: 1643-1651, 2013.

43. Guo C, Chang CC, Wortham M, Chen LH, Kernagis DN, Qin X, Cho YW, Chi JT, Grant GA, McLendon RE, et al: Global identification of MLL2-targeted loci reveals MLL2's role in diverse signaling pathways. Proc Natl Acad Sci USA 109: 17603-17608, 2012.

44. Kim SY, Yang D, Myeong J, Ha K, Kim SH, Park EJ, Kim IG, Cho NH, Lee KP, Jeon JH, et al: Regulation of calcium influx and signaling pathway in cancer cells via TRPV6-Numb1 interaction. Cell Calcium 53: 102-111, 2013.

45. Monteith GR, Davis FM and Roberts-Thomson SJ: Calcium channels and pumps in cancer: Changes and consequences. J Biol Chem 287: 31666-31673, 2012.

46. Yang H, Zhang Q, He J and Lu W: Regulation of calcium signaling in lung cancer. J Thorac Dis 2: 52-56, 2010.

47. Li ZP and Cao Q: Effects of astragaloside IV on myocardial calcium transport and cardiac function in ischemic rats. Acta Pharmacol Sin 23: 898-904, 2002.

48. Zeng L and Zhou MM: Bromodomain: An acetyl-lysine binding domain. FEBS Lett 513: 124-128, 2002 
49. Belkina AC and Denis GV: BET domain co-regulators in obesity, inflammation and cancer. Nat Rev Cancer 12: 465-477, 2012.

50. Dawson MA, Prinjha RK, Dittmann A, Giotopoulos G, Bantscheff M, Chan WI, Robson SC, Chung CW, Hopf C, Savitski MM, et al: Inhibition of BET recruitment to chromatin as an effective treatment for MLL-fusion leukaemia. Nature 478: 529-533, 2011.

51. Greenwald RJ, Tumang JR, Sinha A, Currier N, Cardiff RD, Rothstein TL, Faller DV and Denis GV: E mu-BRD2 transgenic mice develop B-cell lymphoma and leukemia. Blood 103 . 1475-1484, 2004.

52. Belkina AC, Nikolajczyk BS and Denis GV: BET protein function is required for inflammation: Brd2 genetic disruption and BET inhibitor JQ1 impair mouse macrophage inflammatory responses. J Immunol 190: 3670-3678, 2013.

53. Hanahan D and Weinberg RA: Hallmarks of cancer: The next generation. Cell 144: 646-674, 2011.

54. Muñoz-Pinedo C, El Mjiyad N and Ricci JE: Cancer metabolism: Current perspectives and future directions. Cell Death Dis 3 : e248, 2012.

55. Warburg O, Posener K and Negelein E: Ueber den stoffwechsel der tumoren. Biochem Z 152: 319-344, 1924 (In German).

56. Warburg O: On the origin of cancer cells. Science 123: 309-314, 1956.

57. DeBerardinis RJ, Lum JJ, Hatzivassiliou G and Thompson CB: The biology of cancer: Metabolic reprogramming fuels cell growth and proliferation. Cell Metab 7: 11-20, 2008.

58. Han S, Kim S, Bahl S, Li L, Burande CF, Smith N, James M, Beauchamp RL, Bhide P, DiAntonio A, et al: The E3 ubiquitin ligase protein associated with Myc (Pam) regulates mammalian/mechanistic target of rapamycin complex 1 (mTORC1) signaling in vivo through $\mathrm{N}$ - and $\mathrm{C}$-terminal domains J Biol Chem 287: 30063-30072, 2012.

59. Weidensdorfer D, Stöhr N, Baude A, Lederer M, Köhn M Schierhorn A, Buchmeier S, Wahle E and Hüttelmaier S: Control of c-myc mRNA stability by IGF2BP1-associated cytoplasmic RNPs. RNA 15: 104-115, 2009.

60. Nikiforov MA, Chandriani S, Park J, Kotenko I, Matheos D, Johnsson A, McMahon SB and Cole MD: TRRAP-dependent and TRRAP-independent transcriptional activation by Myc family oncoproteins. Mol Cell Biol 22: 5054-5063, 2002.

61. Han S, Witt RM, Santos TM, Polizzano C, Sabatini BL and Ramesh V: Pam (protein associated with Myc) functions as an E3 ubiquitin ligase and regulates TSC/mTOR signaling. Cell Signal 20: 1084-1091, 2008.

62. Yecies JL and Manning BD: Transcriptional control of cellular metabolism by mTOR signaling. Cancer Res 71: 2815-2820, 2011.
63. Zou F, Mao XQ, Wang N, Liu J and Ou-Yang JP: Astragalus polysaccharides alleviates glucose toxicity and restores glucose homeostasis in diabetic states via activation of AMPK. Acta Pharmacol Sin 30: 1607-1615, 2009.

64. Vander Heiden MG, Cantley LC and Thompson CB: Understanding the Warburg effect: The metabolic requirements of cell proliferation. Science 324: 1029-1033, 2009.

65. Zhou G, Myers R, Li Y, Chen Y, Shen X, Fenyk-Melody J, Wu M, Ventre J, Doebber T, Fujii N, et al: Role of AMP-activated protein kinase in mechanism of metformin action. J Clin Invest 108: $1167-1174,2001$.

66. de Krijger I, Mekenkamp LJ, Punt CJ and Nagtegaal ID: MicroRNAs in colorectal cancer metastasis. J Pathol 224 438-447, 2011.

67. Dong H, Lei J, Ding L, Wen Y, Ju H and Zhang X: MicroRNA: Function, detection, and bioanalysis. Chem Rev 113: 6207-6233, 2013.

68. Roy S, Levi E, Majumdar AP and Sarkar FH: Expression of miR-34 is lost in colon cancer which can be re-expressed by a novel agent CDF. J Hematol Oncol 5: 58, 2012.

69. Qiu YY, Hu Q, Tang QF, Feng W, Hu SJ, Liang B, Peng W and Yin PH: MicroRNA-497 and bufalin act synergistically to inhibit colorectal cancer metastasis. Tumour Biol 35: 2599-2606, 2014.

70. Chu Y, Ouyang Y, Wang F, Zheng A, Bai L, Han L, Chen Y and Wang H: MicroRNA-590 promotes cervical cancer cell growth and invasion by targeting CHL1. J Cell Biochem 115: 847-853, 2014.

71. Jiang X, Xiang G, Wang Y, Zhang L, Yang X, Cao L, Peng H, Xue $P$ and Chen D: MicroRNA-590-5p regulates proliferation and invasion in human hepatocellular carcinoma cells by targeting TGF- $\beta$ RII. Mol Cells 33: 545-551, 2012.

72. Yang H,Zheng W,Zhao W, Guan C and An J: Roles of miR-590-5p and miR-590-3p in the development of hepatocellular carcinoma. Nan Fang Yi Ke Da Xue Xue Bao 33: 804-811, 2013 (In Chinese).

73. Fearon ER: Molecular genetics of colorectal cancer. Annu Rev Pathol 6: 479-507, 2011.

74. Efferth T, Li PC, Konkimalla VS and Kaina B: From traditional Chinese medicine to rational cancer therapy. Trends Mol Med 13: 353-361, 2007.

75. Kannaiyan R, Manu KA, Chen L, Li F, Rajendran P, Subramaniam A, Lam P, Kumar AP and Sethi G: Celastrol inhibits tumor cell proliferation and promotes apoptosis through the activation of c-Jun N-terminal kinase and suppression of PI3 K/Akt signaling pathways. Apoptosis 16: 1028-1041, 2011. 